\title{
Skin barrier in rosacea ${ }^{*}$
}

\section{Flavia Alvim Sant'Anna Addor ${ }^{1}$}

DOI: http:/ /dx.doi.org/10.1590/abd1806-4841.20163541

\begin{abstract}
Recent studies about the cutaneous barrier demonstrated consistent evidence that the stratum corneum is a metabolically active structure and also has adaptive functions, may play a regulatory role in the inflammatory response with activation of keratinocytes, angiogenesis and fibroplasia, whose intensity depends primarily on the intensity the stimulus. There are few studies investigating the abnormalities of the skin barrier in rosacea, but the existing data already show that there are changes resulting from inflammation, which can generate a vicious circle caused a prolongation of flare-ups and worsening of symptoms. This article aims to gather the most relevant literature data about the characteristics and effects of the state of the skin barrier in rosacea.
\end{abstract}

Keywords: Cathelicidins; Keratinocytes; Rosacea; Water loss, insensible

\section{INTRODUCTION - GENERAL ASPECTS OF ROSACEA}

Rosacea is a chronic disease, with evolution in outbreaks, characterized by an inflammatory process and vasculopathy. ${ }^{1,2}$

Although its manifestations are varied, usually rosacea is primarily characterized by erythema of several and persistent degree, which worsens suddenly (flushing), with or without papular inflammatory or even pustular lesions, similar to acne, and, in more chronic forms, of telangiectasias. ${ }^{3}$ It occurs mainly on the face and is more prevalent in women, but genetic and ethnic factors may change this relationship between sexes, since it's more common in Caucasians, particularly in the Northern hemisphere.

This fact results in a variable prevalence according to different geographical areas, which can be up to $10 \%$ of population. ${ }^{3}$

Etiology of rosacea appears to be multifactorial: in addition to a genetic predisposition and even to the skin type (being more common in individuals with a family history of rosacea and lower skin types, respectively) that determines an exacerbated and prolonged vascular reaction, intrinsic and environmental factors may encourage vasodilation; these factors, acting alone or in association, lead to the progress of dermatosis, acting as triggering and aggravating factors. ${ }^{4}$

In its natural history, rosacea develops from stimuli that promote vasodilation, such as sun exposure, hot and/or spicy food and drinks, alcohol, physical exercise, high temperature environments or abrupt changes of temperature, besides vasodilators (e.g., antihypertensives) or angiogenic drugs (e.g., topical corticosteroids, topical tretinoin). ${ }^{5,6}$

These stimuli induce episodes of flushing with progressive damage to the endothelium and also angiogenesis, as well as inflammatory changes in the dermis with production of vasoactive substances, and worsening of vascular framework, which will have repercussions in the epidermis. Secondary infections by microorganism of the genre Demodex, especially folliculorum, seem to have a direct association with the present inflammation. ${ }^{7,8}$

There is little consensus in understanding the different manifestations of rosacea, which hinders its classification. Previously classified in 4 evolutionary

Conflict of Interest: None.

Private clinic - São Paulo (SP), Brazil.

C2016 by Anais Brasileiros de Dermatologia 
stages, with variants described, the National Rosacea Society (NRS) developed a classification according to the clinical findings, regardless of evolutionary or pathogenic aspects (erythematotelangiectatic, papulopustular, phymatous and ocular) and only one variant, the granulomatous. ${ }^{9,10}$

Although all studies on rosacea demonstrate the hypersensitivity of the skin to termal, chemical and even biological stimuli (thinking of colonization by Demodex and bacterial infection) there is still no clear correlation between clinical manifestations, damage of the skin barrier (obviously present in any inflammatory dermatosis) and sebaceous activity of the skin.

\section{OBJECTIVE}

This study aims to make a literature review on the state of the skin barrier in rosacea and its role in the etiopathogenesis of this dermatosis as well as the clinical consequences of its changes.

\section{CHARACTERISTICS OF SKIN BARRIER IN RO- SACEA AND ETIOPATHOGENESIS}

\section{Sebum production}

Rosacea may occur in patients with dry skin or skin with important seborrheic levels, not being necessarily linked to a skin type. Studies assessing some parameter of the skin barrier in patients with rosacea are very scarce. While most studies have focused on sebum secretion, some authors point to conditions that occur with dry skin.

It is known that lipids produced by sebaceous glands are responsible for three-dimensional organization of skin surface lipids, contributing to the integrity of its barrier. These lipids also exhibit strong antimicrobial activity, besides carrying antioxidant molecules to the surface and expressing proinflammatory and anti-inflammatory properties, while maintaining the $\mathrm{pH}$ slightly acidic. ${ }^{11}$

A recent study evaluated 135 patients with rosacea and without treatment, and showed that the erythematotelangiectatic form of the disease is more common in dry skin than in seborrheic skin. ${ }^{12}$

Papulopustular form was evaluated in another work, regarding the sebum level, against a control group: sebaceous levels were normal, but with a significant reduction in hydration levels. Even after treatment with oral minocycline, despite the clinical improvement, there was no significant change in the amount of sebum found. ${ }^{13}$

There is no study, however, relating the clinical forms of rosacea with sebaceous levels.

Also regarding the study of lipids from the sebaceous glands, it showed an imbalance in the fatty acid concentration in sebum in patients with papulopustular rosacea: myristic acid was present in higher con- centration in sebum of these patients compared with a control group without the dermatosis; while saturated long-chain fatty acids (behenic, tricosanoic and lignoceric), as well as monounsaturated cis-11-eicosanoic, were in lower concentrations compared with the control group..$^{14}$

These data support the evidence that the sebum production, in particular of fatty acids, may influence the barrier integrity of patients with papulopustular rosacea.

Demodex infestation, a known aggravating factor of inflammation in rosacea, seems to be more common in patients with barrier changes; a study on facial skin diseases where there is a prevalence of Demodex (perioral dermatitis, seborrheic dermatitis, steroid dermatitis and rosacea itself) demonstrated higher levels of infestation in drier or more seborrheic skin, when compared with skin with normal levels of hydration and sebum secretion. ${ }^{15}$ Demodex also predominates in patients over 30 years.

\section{Inflammatory changes}

Rosacea is a dermatosis with complex physiopathology; these patients exhibit a modified innate immune response to environmental stimuli, favoring inflammation. Over time, these inflammatory outbreaks lead to dermal fibrotic conditions. However, not all patients with erythematotelangiectatic form necessarily progress to phymatous or papulopustular form. ${ }^{16}$

The innate response involves the secretion of antimicrobial peptides (AMP), from the keratinocytes, sebocytes and mast cells. Expression of these peptides is strictly regulated, because although it is an important line of defense, it can also cause tissue damage.

Among AMPs, the group of cathelicidins has received special attention in the physiopathology of rosacea.

Cathelicidins are a family of cationic AMPs, of the same class of defensins and histatins, found in macrophages and polymorphonuclear leukocytes. ${ }^{17}$

In healthy skin, this AMP is little detected in keratinocytes; on the other hand, during an infection or damage there is a major production of cathelicidins. In the skin with rosacea, there is a higher concentration of cathelicidins; in normal skin, this AMP is processed by proteases of the kallikrein family, in particular 5 and 7 , which results in another peptide, cathelicidin LL-37, produced only in humans. ${ }^{18}$

Cathelicidin LL-37 is an important molecule in the innate metabolism, and inflammatory diseases such as rosacea, there is a disturbance in its processing, which results in peptide fragments that cause inflammation, erythema, and even telangiectasia, as shown in figure 1. 19,20

Environmental factors that trigger cathelicidins 
increasing in rosacea are:

- UV-radiation: the synthesis of vitamin D caused by UVB radiation induces expression of cathelicidins in keratinocytes.

- Presence of Demodex mites: chitin protein from these microorganisms activates the inflammatory response in keratinocytes and binds the presence of mites with the increasing of protease activity and the cathelicidins induced inflammation. ${ }^{21}$

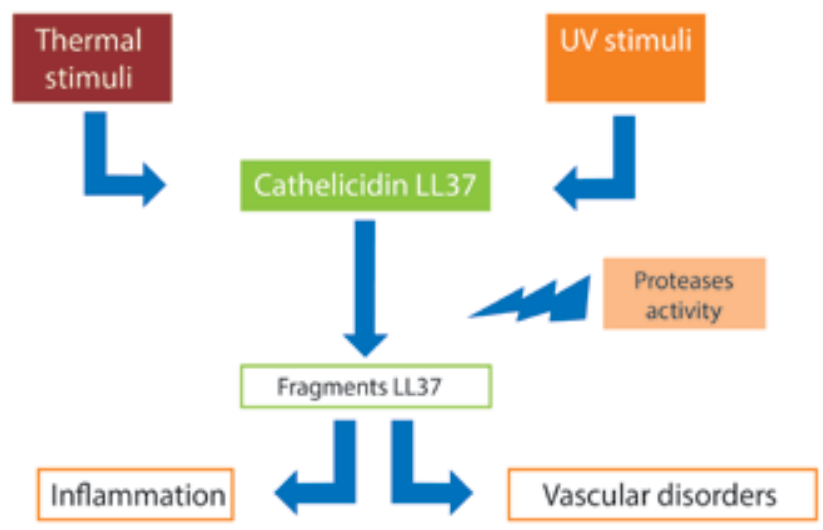

Figure 1: Role of cathelicidin 37 in the pathogenesis of rosacea. Environmental stimuli increase expression of cathelicidin 37, whose fragments resulting from protease activity stimulate the inflammatory process and induce vascular disorders in individuals with rosacea

Epidermal inflammation and cathelicidins higher concentration leads to lipid synthesis disruption and stratum corneum formation, affecting the barrier function and, concomitantly, favoring the classic symptoms of rosacea, such as itching, burning and stinging. ${ }^{22}$

\section{Vascular changes}

Medium facial erythema is the main sign of rosacea, and is present in all clinical forms, being virtually its first manifestation.

In outbreaks progressively intense and frequent, it presents uncomfortable symptoms to the patient such as warmth, stinging and burning of the skin.

Vasodilation that causes erythema, as well as vascular changes that are installed with the development of dermatosis, such as telangiectasias, elapses of a modified vasoactive response, from a neurovascular dysfunction. ${ }^{23}$

These changes lead to an increased blood flow, which generates the sensation of warmth, particularly in the most affected areas.
As described above, cathelicidin LL-37 is strongly increased in the affected area of skin in rosacea. This peptide has proinflammatory action and an "alarm" function, affecting vascular growth, thus leading to angiogenesis and neovascularization. ${ }^{20}$

In patients with the erythematoustelangiectatic form, there is a strong presence of proinflammatory and vasoregulator genes, even in the earliest forms of the disease. A predominantly perivascular inflammatory infiltrate also occurs, consisting of lymphocytes, macrophages and mast cells, also with increased vascular endothelial growth factor (VEGF). ${ }^{24}$

The lymphatic involvement is also present and is important in rosacea, especially in papulopustular and phimatous forms.

VEGF also acts in the expansion of lymphatic vessels, but there is no evidence of lymphangiogenesis. $^{25}$

The increased vascularity and dermal vasodilation contribute to an increased transepidermal water loss, which acts as stimuli to keratinocyte proliferation and differentiation. ${ }^{26}$

\section{Neural changes}

There is a neural interaction with the immune and vascular changes, triggered by factors such as spicy foods and emotional stress.

Physiologically, the dermal subpapilar plexus contains smaller vessels including venules, arterioles and capillaries; more deeply, larger vessels are found, which are primarily modulated by sympathetic innervation (adrenergic). ${ }^{27}$

Local changes in temperature lead to vasodilation or vasoconstriction exacerbated reactions, in addition to a stimulus induced by alpha adrenergic receptors. ${ }^{28}$

Endothelial cells activated in the affected area express several adhesion molecules and cytokine receptors, facilitating activation and leukocyte migration, thus exacerbating inflammation. ${ }^{16}$

More recently, studies present evidence that TRPV1 (transient receptor potential cation channel 1) receptor, sensitive to capsaicin, has higher expression in patients with rosacea, aiding the explanation of greater discomfort to chemically similar substances in this compound, as some perfumes and herbal extracts. ${ }^{29,30}$

\section{Rosacea and "sensitive skin"}

All phenomena described above act synergistically in the perpetuation of perivascular, dermal and epidermal inflammation. This process induces an acceleration of epidermal proliferation and differentiation, resulting in a functionally deficient stratum corneum, with low ability to attract and retain water. These findings enhance transepidermal water loss, 
which is already increased by the underlying inflammation, exacerbating the reduction of the water portion in the skin and worsening its barrier function, as shown in Figure 2. ${ }^{31}$

The $\mathrm{pH}$ of the skin surface also undergoes changes with the alterations in the stratum corneum, with an increase and consequent loss in antimicrobial function.

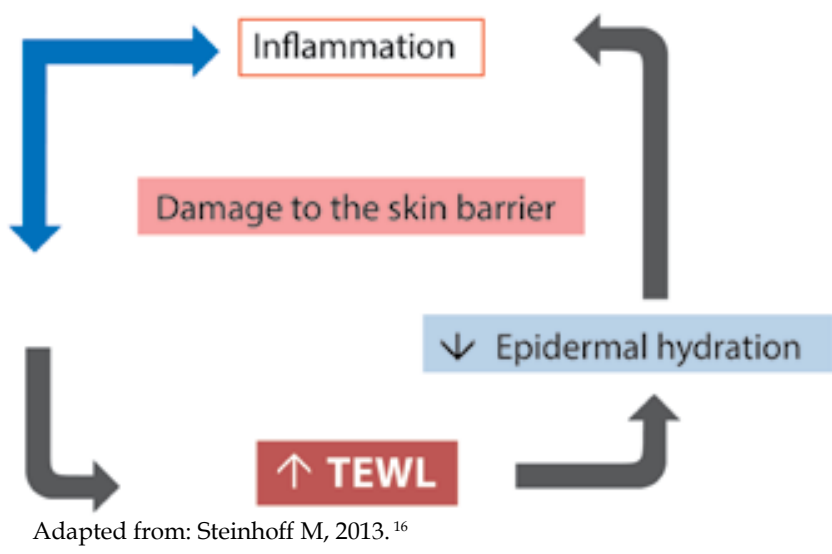

FIGURE 2: Relationship between damage of the skin barrier and skin inflammation. The inflammatory process reduces the barrier function; allied to vasodilation, also present in rosacea, occurs an increase in transepidermal water loss (TEWL) with consequent reduction of epidermal hydration levels, constituting a stimulus to inflammation

The described changes are translated clinically by a lower tolerance to topical products such as soaps, hot water, low $\mathrm{pH}$ substances (lactic acid, retinoic acid, etc.), resulting in burning sensations, itching, and stinging, possibly with a decreased in the irritative threshold known as "sensitive skin". ${ }^{32}$ This condition is often described in patients with rosacea.

A study with biophysical measurements in patients with papulopustular rosacea showed that, when compared with normal individuals, sebum levels are almost normal, but there is a significant reduction in hydration levels and a rise in $\mathrm{pH}$ in centrofacial region..$^{33}$

One of the largest studies in rosacea patients was conducted in 1997 by the National Rosacea Society involving 1023 patients; of these, 82\% reported irritable skin, with burning or stinging sensation, when using cosmetic products, especially those with the greatest potential to interfere with the skin barrier, such as exfoliating, soaps and tonics. ${ }^{34}$
This hypersensitivity is at least partially explained by a barrier previously damaged. The barrier damage been demonstrated in a sample of patients by the increasing of transepidermal water loss (TEWL) both in papulopustular and erythematoustelangiectatic forms. ${ }^{22}$

In addition to increased TEWL and hydration loss, there is a change in the innate immune functions, leading to an increase of AMPs, including cathelicidin. These phenomena are not only associated, but also co-regulated. ${ }^{35}$

Some recent evidences show that, in the injured normal skin, or even in the skin with irritant dermatitis, there is a tendency to reduce the expression of kallikrein, including 5 and 7. ${ }^{36,37}$

In rosacea, there is evidence that the pattern of the innate immune response is altered, with increased expression of TLR2 receptor (toll-like receptor 2), resulting in greater expression of kallikrein 5 from keratinocyte. ${ }^{38-40}$

The barrier damage observed with rosacea possibly increases even more due to the higher expression of kallikrein, which would usually be normal or even decreased in other inflammatory dermatosis. ${ }^{41}$

This perpetuation of the barrier dysfunction, clinically translated by dry or scaly skin, must also be taken into consideration in the treatment of rosacea. ${ }^{42}$

Some studies have shown that inadequate topical products may worsen rosacea, as well as proper care can help restore the skin barrier, helping to control the symptoms and the inflammation. ${ }^{42,43}$ Use of moisturizing formulations that increase hydration levels reduces the damage to the stratum corneum proteins and the removal of epidermal lipids, which can provide physiological elements to repair the skin barrier, and do not contain substances with known irritation potential, helping inhibit the inflammatory process and, hence, the symptomatology. ${ }^{43-45}$

\section{CONCLUSION}

Rosacea is a complex dermatosis, with inflammatory, neural and vascular mechanisms, in which environmental factors can interfere negatively or positively in its evolution. Improvement of the barrier function can significantly reduce symptoms, since, besides helping to reduce inflammation, it can decrease the interaction with substances with irritate potential. These two factors are fundamental in improving the symptomatology of patients with rosacea.] 


\section{REFERENCES}

1. Berg M, Lidén S. An epidemiological study of rosacea. Acta Derm Venereol. 1989;69:419-23

2. Bonamigo RR. Rosacea: risk factors, etiology and pathogenesis. An Bras Dermatol 1999;74:621-4

3. Wilkin JK. Rosacea. Pathophysiology and treatment. Arch Dermatol. 1994;130:359-62.

4. Berg M, Lidén S. An epidemiological study of rosacea. Acta Derm Venereol. 1989;69:419-23.

5. Crawford GH, Pelle MT, James WD. Rosacea: I. Etiology, pathogenesis, and subtype classification. J Am Acad Dermatol. 2004;51:327-41.

6. Buechner AS. Rosacea: An Update. Dermatology. 2005;210:100-8.

7. Jarmuda S, O'Reilly N, Zaba R, Jakubowicz 0, Szkaradkiewicz A, Kavanagh K. Potential role of Demodex mites and bacteria in the induction of rosacea. $\mathrm{J}$ Med Microbiol. 2012;61:1504-10.

8. Forton FM. Papulopustular rosacea, skin immunity and Demodex: pityriasis folliculorum as a missing link. J Eur Acad Dermatol Venereol. 2012;26:19-28.

9. Jansen T. Clinical presentations and classification of rosacea. Ann Dermatol Venereol. 2011;138:S192-200.

10. Jansen T, Plewig G. Rosacea: classification and treatment. J R Soc Med. 1997;90:144-50.

11. Zouboulis CC. Acne and sebaceous gland function. Clin Dermatol. 2004;22:360-6.

12. Tan J, Blume-Peytavi U, Ortonne JP, Wilhelm K, Marticou L, Baltas E, et al. An observational cross-sectional survey of rosacea: clinical associations and progression between subtypes. Br J Dermatol. 2013;169:555-62.

13. Ní Raghallaigh S, Powell FC. Epidermal hydration levels in rosacea patients improve after minocycline therapy. Br J Dermatol. 2014;171:259-66.

14. Ní Raghallaigh S, Bender K, Lacey N, Brennan L, Powell FC. The fatty acid profile of the skin surface lipid layer in papulopustular rosacea. $\mathrm{Br} \mathrm{J}$ Dermatol. 2012;166:279-87.

15. Zhao YE, Peng Y, Wang XL, Wu LP, Wang M, Yan HL, et al. Facial dermatosis associated with Demodex: a case-control study. J Zhejiang Univ Sci B. 2011:12:1008-15

16. Steinhoff M, Schauber J, Leyden JJ. New insights into rosacea pathophysiology: A review of recent findings. J Am Acad Dermatol. 2013;69:S15-26.

17. Wang G. Human antimicrobial peptides and proteins. Pharmaceuticals (Basel). 2014;7:545-94.

18. Yamasaki K, Di Nardo A, Bardan A, Murakami M, Ohtake T, Coda A, et al. Increased serine protease activity and cathelicidin promotes skin inflammation in rosacea. Nat Med. 2007;13:975-80.

19. Yamasaki K, Schauber J, Coda A, Lin H, Dorschner RA, Schechter NM, et al. Kallikrein-mediated proteolysis regulates the antimicrobial effects of cathelicidins in skin. FASEB J. 2006;20:2068-80.

20. Reinholz M, Ruzicka T, Schauber J. Cathelicidin LL-37: An Antimicrobial Peptide with a Role in Inflammatory Skin Disease. Ann Dermatol. 2012;24:126-35.

21. Dirschka T, Tronnier H, Fölster-Holst R. Epithelial barrier function and atopic diathesis in rosacea and perioral dermatitis. Br J Dermatol. 2004;150:1136-41.

22. Torok HM. Rosacea skin care. Cutis. 2000;66:14-6.

23. Del Rosso JQ. Advances in Understanding and Managing Rosacea: Part 1.Connecting the Dots Between Pathophysiological Mechanisms and Common Clinical Features of Rosacea with Emphasis on Vascular Changes and Facial Erythema J Clin Aesthet Dermatol. 2012;5:16-25.

24. Steinhoff M, Buddenkotte J, Aubert J, Sulk M, Novak P, Schwab VD, et al. Clinical,cellular, and molecular aspects in the pathophysiology of rosacea. $J$ Investig Dermatol Symp Proc. 2011;15:2-11.

25. Schwab VD, Sulk M, Seeliger S, Nowak P, Aubert J, Mess C, et al. Neurovascular and neuroimmune aspects in the pathophysiology of rosacea. J Investig Dermatol Symp Proc. 2011;15:53-62.

26. Voegeli R, Rawlings AV, Doppler S, Schreier T. Increased basal transepidermal water loss leads to elevation of some but not all stratum corneum serine proteases. Int J Cosmet Sci. 2008;30:435-42.

27. Schwab VD, Sulk M, Seeliger S, Nowak P, Aubert J, Mess C, et al. Neurovascular and neuroimmune aspects in the pathophysiology of rosacea. J Investig Dermatol Symp Proc. 2011;15:53-62.

28. Johnson JM, Kellogg DL Jr. Local thermal control of human cutaneous circulation. J Appl Physiol (1985). 2010;109:1229-38.

29. Nilius B, Owsianik G, Voets T, Peters JA. Transient receptor potential cation channels in disease. Physiol Rev. 2007;87:165-217.

30. Sulk M, Seeliger S, Aubert J, Schwab VD, Cevikbas F, Rivier M, et al. Distribution and expression of non-neuronal transient receptor potential (TRPV) ion channels in rosacea. J Invest Dermatol. 2012;132:1253-62.
31. Tagami H, Kanamaru Y, Inoue K, Suehisa S, Inoue F, Iwatsuki K, et al.Wate sorption-desorption testo $f$ the skin in vivo for functional assessment of the stratum corneum. J Invest Dermatol. 1982;78:425-8.

32. Lev-Tov H, Maibach HI. The sensitive skin syndrome. Indian J Dermatol. 2012;57:419-23.

33. Ní Raghallaigh S, Bender K, Lacey N, Brennan L, Powell FC. The fatty acid profile of the skin surface lipid layer in papulopustular rosacea. Br J Dermatol. 2012;166:279-87.

34. Dirschka T, Tronnier H, Fölster-Holst R. Epithelial barrier function and atopic diathesis in rosacea and perioral dermatitis. Br J Dermatol. 2004;150:1136-41.

35. Elias PM. The skin barrier as an innate immune element. Semin Immunopathol. 2007;29:3-14.

36. Xie H, Li L, Xiong LD, Liao F, Zhang GR. [The changes of skin barrier of patients with different facial dermatitis and the comparison of CE and KLK5].Sichuan Da Xue Xue Bao Yi Xue Ban. 2013;44:940-4, 998.

37. de Koning HD, van den Bogaard EH, Bergboer JG, Kamsteeg M, van VlijmenWillems IM, Hitomi K, et al. Expression profile of cornified envelope structural proteins and keratinocyte differentiation-regulating proteins during skin barrier repair. Br J Dermatol. 2012;166:1245-54.

38. Yamasaki K, Kanada K, Macleod DT, Borkowski AW, Morizane S, Nakatsuji T, et al. TLR2 expression is increased in rosacea and stimulates enhanced serine protease production by keratinocytes. J Invest Dermatol. 2011;131:688-97.

39. Meyer-Hoffert U, Schröder JM. Epidermal proteases in the pathogenesis of rosacea. J Investig Dermatol Symp Proc. 2011;15:16-23.

40. Coda AB, Hata T, Miller J, Audish D, Kotol P, Two A, et al. Cathelicidin, kallikrein 5 and serine protease activity is inhibited during treatment of rosacea with azelaic acid 15\% gel. J Am Acad Dermatol. 2013;69:570-7.

41. Del Rosso JQ. Adjunctive skin care in the management of rosacea: cleansers moisturizers, and photoprotectants. Cutis. 2005;75:17-21.

42. Levin J, Miller R. A Guide to the Ingredients and Potential Benefits of Over-theCounter Cleansers and Moisturizers for Rosacea Patients. J Clin Aesthet Dermatol. 2011;4:31-49.

43. Rawlings AV, Harding CR. Moisturization and skin barrier function. Dermatol Ther. 2004;17:43-8.

44. Pelle MT, Crawford GH, James WD. Rosacea: II. therapy. J Am Acad Dermatol. 2004:51:499-512.

45. Loden M. Role of topical emollients and moisturizers in the treatment of dry skin barrier disorders. Am J Clin Dermatol. 2003:4:771-88.

\author{
MAILING ADDRESS: \\ Flavia Alvim Sant'Anna Addor \\ Rua Attilio Delanina, 178 \\ Campesina \\ 0623-110 - Osasco - SP \\ Brazil \\ Email:flavia@medcinonline.com.br
}

How to cite this article: Addor FAS. Skin barrier in rosacea. An Bras Dermatol. 2016;91(1):59-63. 